\title{
Design of Heterogeneous Data Exchange Technology for Teaching Resources Based on ICMPv6
}

\author{
https://doi.org/10.3991/ijet.v13i11.9600 \\ Wei Fang \\ Huaibei Vocational and Technical College, Anhui, China \\ weifang $3482 \mathrm{C} 21 \mathrm{cn} . \mathrm{com}$
}

\begin{abstract}
To promote the innovation of teaching resources and heterogeneous data exchange platform technologies and theories, heterogeneous data exchange platforms based on ICMPv6 teaching resources were studied. First, based on ICMPv6, the middle-tier architecture of the heterogeneous data exchange platform for teaching resources was studied. Second, the application layer architecture in the heterogeneous data exchange platform system of educational resources was studied. The middle layer and application layer were designed and implemented. Finally, the system was applied to the education platform to reflect its performance. The results showed that the ICMPv6 system could solve data exchange and data sharing systems between schools and homes. Contributions were made in solving interactive education between home and school. To sum up, it is feasible to use ICMPv6 on heterogeneous educational resources exchange platform.
\end{abstract}

Keywords-ICMPv6, heterogeneous data exchange platform, teaching resources, architecture

\section{$1 \quad$ Introduction}

The update of network technology improves the performance of the network. Users and network applications are gradually increasing, and the demand for big data technologies and applications is also constantly evolving, which has promoted the innovation of management information platforms in various industries. As the basic carrier of informatization and big data application, the data exchange platform has been widely concerned by industry and scholars. The data exchange platform is an important platform for heterogeneous data release, integration, and conflict handling. In addition, it provides the foundation for a richer data analysis application for the upper layer. In response to the development of this social environment, to promote the innovation of data exchange platform technology and theory, the State Council issued the "National Medium- and Long-Term Science and Technology Development Plan." In the outline, modern service industry information support technology and large-scale application software are the current priority projects. In particular, data exchange platform technology is a typical representative of service industry information support technology. At the same time, in response to the policy, the National Natural Science Foundation of 
China also gave support to research funds in this area. Therefore, the research of data exchange platform foundation and core technology has important theoretical significance. Research and discussion on data exchange platform is a hot topic at home and abroad.

At present, the informatization process in various fields is relatively fast, and the research environment is very good. However, the research process cannot be smooth sailing. The heterogeneity of different environments, the heterogeneity of data formats, and the heterogeneity of database types lead to various problems in the process of information integration. The efficiency of data sharing is affected to varying degrees. Thus, research on the architecture of the data exchange platform is necessary. Regarding the integration of heterogeneous data, each node is transmitted and exchanged on the data exchange platform. However, the data transmission and exchange efficiency of the entire data sharing platform cannot keep up with the exchange speed. The efficiency and security of data exchange platform need to be improved. At the same time, the application of the data exchange platform has been continuously promoted. Exchange platform applications based on various data are emerging one after another. The data will be analyzed using ICMPv6.

In summary, based on the educational resource data exchange platform, the architecture of the data exchange platform is studied. The efficiency and security of the data exchange platform is studied. The intelligent guidance application scenario based on the data exchange platform is studied. The purpose is to provide a technological and theoretical basis for the subsequent teaching resources heterogeneous data exchange platform.

\section{State of the art}

Aiming at the end-to-end performance evaluation of data exchange platform in data transmission process, according to the network characteristics of education resource data exchange platform, an end-to-end bottleneck traffic measurement method based on ICMPv6 is proposed. Tracert is used to record all router addresses and hop counts on the end-to-end path. The IPv6 flow label is used to extract information about the ICMPv6 timeout packet and its corresponding router address and timestamp. Finally, the subtraction method is used to calculate the end-to-end bottleneck flow. Simulation results show that the link bandwidth of the bottleneck bandwidth measurement method based on the subtraction rule is stable at $20 \%$, which can effectively improve the link utilization.

Bartoli et al. [1] introduced a method for database replication and segmentation of a large number of commercial and academic database management systems using Java and Hibernate mapping. Cai et al. [2] analyzed CSCW, XML, JDBC and heterogeneous databases. With the metadata of the database, a solution for middleware and LDAP directory service heterogeneous databases was proposed. González et al. [3] pointed out that IBM's software research and development laboratory research (China department) proposed an improved solution, namely WAS (Web Sphere Application Server) and WBIC (Web Sphere Business Integration Connect) based on distributed basis. The 
solution provides a flexible environment for information exchange via the Rosetta Net XML specification. Hakiri et al. [4] proposed a new technique for integrating the Heterogeneous Extensible Markup Language (XML) model, which is called XDEHD. The returned media mode contains all the concepts and relationships of the source, and they are not duplicated. The detailed technique is divided into three steps: First, all subpatterns are extracted from the source by decomposing the pattern source. Each submode contains three levels, which are ancestors, roots, and leaves. Second, the technique matches and compares the sub-modes and returns the relevant candidate submodes. A semantic proximity function is implemented to measure the degree of modeling of the concept of the submodel in the source. Finally, by integrating candidate subpatterns, new patterns are created. The smallest and complete unified mode is obtained. The correlation strength function was developed to be calculated among candidate sub-patterns in all data sources. The element repeat function is used to calculate each element that is repeated between candidate sub-patterns. Hussain et al. [5] pointed out that the bottleneck bandwidth measurement methods of the educational resource data exchange platform are mainly divided into two types: the bottleneck bandwidth detection method based on Pathchar and the bottleneck bandwidth detection method based on packet pairing. Karkazis et al. [6] studied the bottleneck bandwidth detection method based on Pathchar. The main principle of this method is to use ICMP (Internet Control Message Protocol) to calculate the round-trip time of packet grouping. The time of each hop is calculated by comparing the number of bits of the packet and the roundtrip time. The round-trip time (RTT) consists of three parts. They are the sending time of packets, the transmission time of packets and the waiting time of packets. The transmission time of the data packet is calculated as $\mathrm{S} / \mathrm{\rho}$. $\mathrm{S}$ is the length of the data packet to be sent, and $\rho$ is the bandwidth of the link. The packet propagation time refers to the round-trip time of the signal used to transmit data in the link, which is generally related to the speed of light. Jourjon et al. [7] pointed out that the waiting time of a packet refers to the waiting time in the buffer before the packet is sent. It is mainly generated by the resource contention process of packets in the intermediate routers and switches. $\mathrm{Xu}$ et al. [8] studied the internal acceleration buffer crossbar switch. The cost of the crosspoint buffer requires at least $\mathrm{O}(\mathrm{c} \cdot \mathrm{N} 2)$ of memory space. $\mathrm{c}$ is the number of bits in the cell, which is used to reduce the complexity of the control. The circuit of the crosspoint buffer and the scheduler occupies a large area of the chip, which also severely limits the scalability of the buffer crossbar.

Although domestic and foreign scholars and related network technologies have designed many methods to detect network bandwidth, the accuracy of network bandwidth detection is still a key research issue in the scientific community. Traditional parameters for evaluating network bottleneck bandwidth include link bandwidth, available bandwidth, bottleneck bandwidth, and block transmission bandwidth. Common protocols that can detect network bandwidth include SNMP (Simple Network Management Protocol), which allows network administrators to directly read network information. However, the biggest limitation of this approach is that it is not suitable for end-to-end end customers. Only administrators can get bandwidth information. The end user of the data platform cannot detect the bandwidth information of the data platform in real time. 
In summary, the main research method detects the characteristics of IPv6 packets. According to the subtraction model, the asymmetric packet in the IPv6 data packet is detected to achieve the purpose of detecting the traffic. For the users of common data platforms, a new end-to-end bottleneck bandwidth monitoring method is designed. Compared with previous studies, this method can achieve end-to-end bottleneck bandwidth measurement without additional equipment.

\section{$3 \quad$ Methodology}

\subsection{An overview of ICMPv6}

ICMPv6 (Internet Control Managemet Protocol Version 6) is an Internet control information protocol developed for use with IPv6. Like IPv4, IPv6 also needs to use ICMP, and the old version of ICMP cannot meet all the requirements of IPv6. Therefore, a new version of ICMP was developed, which is called ICMPv6. The Internet control information protocol is an important part of the IP protocol. ICMPv6 reports to the source node the error and information about the destination address transmission of the IPv6 packet. It has features such as error reporting, network diagnostics, neighbor discovery, and multicast implementation. In IPv6, ICMPv6 implements ICMP, ARP, and IGMP functions in IPv4.

In addition to the basic functions of IPv4 ICMP, ICMPv6 also includes the following two functions:

Multicast Listener Discovery (MLD): This protocol completes the management of multicast members in a subnet. The MLD protocol defines three ICMPv6 messages: Multicast Listening Query Message: The multicast router sends this message to the multicast listener in the subnet to obtain the status of the multicast listener. Multicast Listener Report Message: The multicast listener reports the status to the multicast router, including leaving a multicast group.

Neighbor Discovery: The Neighbor Discovery Protocol implements IPv6 address resolution protocols. The ICMPv6 router discovery protocol and ICMPv6 redirect message are used to manage communication between nodes on the same link.

The agreement defines five ICMPv6 messages:

Router Advertisement Message: The router sends it to the link in multicast mode to announce its availability and its associated configuration parameters. There are two ways to send the message. One is unsolicited, periodic router advertisements, and the other is requested router advertisements. After receiving the router's request from the host, it is sent out as a response. Router request message: The message is sent by the host to the local router, asking it to immediately send a router advertisement message. Neighbor request message: The node sends a neighbor solicitation message to request the link-layer address of the neighbor to verify the reachability and uniqueness of the neighbor link-layer address. Neighbor notification message: When receiving the neighbor request message or the link layer address changes, the node sends a neighbor advertisement message to announce its link address information to the neighbor node. 
Redirect message: The router sends a redirect message to tell the host to redirect the path.

\subsection{Two methods of measuring the bottleneck bandwidth}

Bandwidth detection method based on Pathchar: The main principle of this method is to use ICMP (Internet Control Message Protocol) to calculate the round-trip time of packet grouping, and calculate the time of each hop by comparing the number of bits of the packet and the round-trip time. The round-trip time (RTT) consists of three parts: packet sending time, packet transmission time and packet waiting time. The transmission time of the data packet is calculated as $S / \rho$. $S$ is the length of the data packet to be transmitted, and $\rho$ is the bandwidth of the link. The packet propagation time refers to the round-trip time of the signal used to transmit data in the link, which is generally related to the speed of light. The waiting time of a data packet refers to the waiting time in the buffer before the data packet is sent, which is mainly generated by the data contention process of the data packet in the intermediate router switch.

Detection method based on packet pair: A packet pair refers to a packet grouping consisting of two packets. The principle is shown in Figure 1. The probe packet is $\mathrm{N}$ probe packets, which can be expressed as $\{0 \mathrm{P}, 1 \mathrm{P}, \ldots, \mathrm{n} 1 \mathrm{P}\}$. The probe packet size is $\mathrm{S}$. The bottleneck bandwidth is $\mathrm{C}$. ( $\mathrm{t}$ ) is the time interval between the first packet and the last packet at the bottleneck. Therefore, the calculation formula of the bottleneck bandwidth $\mathrm{C}$ is $\mathrm{C}=(\mathrm{N}-1) \mathrm{S} / \mathbf{\Delta}(\mathrm{N})$. The performance of the packet-to-measurement data exchange platform bandwidth value is much better than the rest of the packet combination. Schematic diagram of a monitoring method based on packet pairs is shown in Figure 1.

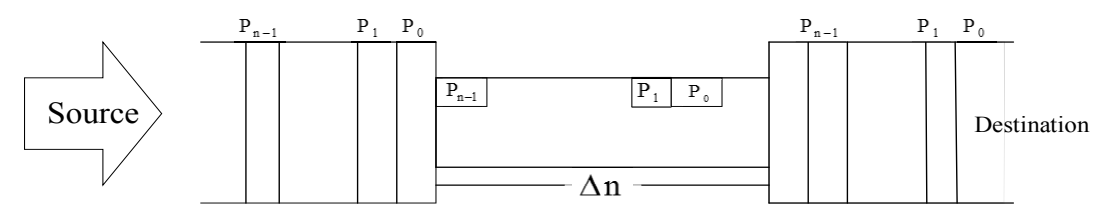

Fig. 1. Schematic diagram of a monitoring method based on packet pairs

\subsection{Application of ICMPv6}

Long measurement time of pathchar based on IPv4 protocol may lead to inaccurate measurement. However, one advantage of pathchar is that it can perform bandwidth measurement operations at any node. Therefore, the IPv6 protocol is applied to redesign how to measure network bottleneck traffic using the characteristics of the pathchar algorithm under the IPv6 protocol. The starting point is to take advantage of the new features of the ICMPv6 protocol. The packet pairing method based on the subtraction rule performs bottleneck flow measurement on the IPv6-based data exchange platform. The specific principles are as follows: 
Step1: The tracert method is used to record all router addresses that the packet is sent from the source host to the destination host, and the hop count $\mathrm{n}$ is calculated. This path is used as the path for bottleneck traffic testing of the data exchange platform.

Step2: According to the path measured by tracert, the transmission path between the source host and the destination host is fixed by using the IPv6 flow label. In this way, the remaining packets will be transmitted along a unique path, that is, the transmission path of the packet is the same. The statistical hop value $\mathrm{n}$ is written as a hop limit field to the IPv6 header. By using the hop limit, ICMPv6 automatically generates timeout packets, reports them to the source host and discards these timeout packets.

Step3: The final result is calculated using the subtraction model, which avoids the synchronization of the host and does not affect the normal transmission of other data streams.

The principle of the data platform bottleneck bandwidth detection method based on the subtraction model is shown in Figure 2. Node A represents the source host, and node $\mathrm{B}$ represents the destination host. The source host to the destination host consists of an n-hop router. Assuming that the path consisting of source node A, destination node $\mathrm{B}$, and $\mathrm{n}$-hop router is $\mathrm{r}$, then the number of nodes on $\mathrm{r}$ is $\mathrm{n}+1$, and $\mathrm{iN}$ and $\mathrm{ilN}$ are called adjacent nodes. Il is the forwarding link existing between the node iN and i1N, and the link bandwidth is iC. The principle of the detection method is shown in Figure 2.

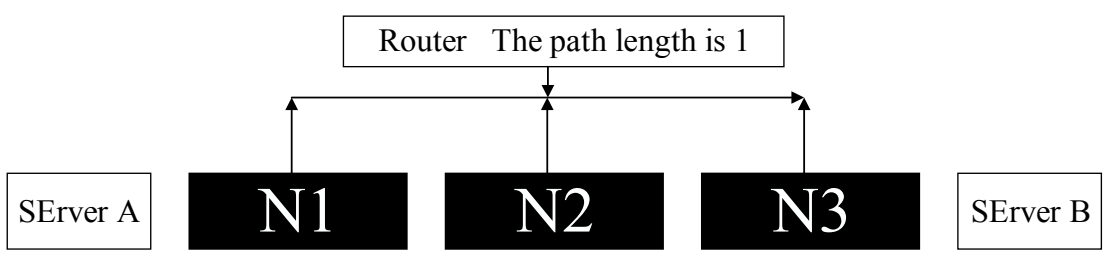

Fig. 2. Schematic diagram of the data platform bottleneck bandwidth detection method of the subtraction model

According to such a data graph, the principle of measuring the bottleneck bandwidth of the data exchange platform is as follows:

$$
t_{i}^{k}=t_{0}^{k}+\sum_{i=0}^{t=1}\left(\frac{s^{k}}{c^{i}}+\frac{d^{i}}{v^{i}}+q_{i}^{k}\right)
$$

The design premise of the proposed algorithm mainly depends on the new features of the IPv6 protocol. Compared with the traditional IPv4 protocol, IPv6 designs data in the same transport stream as the same stream label. Therefore, the transmission path of a series of test streams will be uniquely fixed. There is no problem that different packets appearing in the IPv4 protocol are transmitted over different paths. This feature of IPv6 uniquely determines the path during packet transmission. The inclusion theory analyzed in the relevant work is completely established in the IPv6 protocol. In this algorithm, time compression and expansion problems are generated at the bottleneck of the link. 
To avoid the shortcomings of the traditional package, the subtraction rule is used to reduce the impact of packet queuing problems on bottleneck bandwidth measurement. In addition, the asymmetric packet monitoring method based on the subtraction model is closer to the actual network traffic, the packet size can be inconsistent, and background flow interference is allowed. Therefore, the measurement result is relatively accurate, which can effectively reduce the measurement test and reduce the overhead caused by the bottleneck bandwidth measurement on the actual network. Compared with the traditional IPv4 design method, the flow label used by the IPv6 protocol makes the bottleneck bandwidth test based on the packet and column data simpler and easier to operate. It helps to improve the efficiency of network transmission.

\subsection{Experimental verification}

This data conversion platform can support multi-user use of the home school interactive education platform. The front end contains two very important information tables: the source data table and the conversion table. The source data table holds the task data shared between different systems and the converted data, and the conversion table is used to save the changed data. The conversion table makes the task of sending change data easier and increases the security of the system. At the same time, it can reduce the number of system crashes and is easy to recover even after a crash, to minimize data loss. The conversion table is equivalent to the log of the database, but it is a log table for the condition. If the system crashes, the data is manipulated by the content recorded in the conversion table to recover the data, thereby reducing the loss of data.

The data conversion platform mainly includes the following functions:

Data extraction. The central database contains several task tables: transfer tables, mapping tables, and receive tables. When the data shared by a task system changes, the system will capture the changed data and send it to the conversion table. The task's scheduler wraps the changed data into data in the form of ICMPv6, while sealing and sending it to the central database.

Data conversion. The data version change process includes two conversions: The first time is to convert a relational database into an incomplete relational database. Another time is to convert an incomplete relational database into a relational database. The source and target databases may be different databases with different data structures and data formats. The database must have a uniform standard that makes conversion easy, and this standard is based on the standards of the Ministry of Education. The data format of the source database was changed to the format of the central database. Then, the data format of the central database is changed to the data format of the target database. Throughout this process, the format of the data is in ICMPv6 format.

Data transmission. Through the front end, the parameters of the shared database are set to the corresponding service system. The WEB service request is then sent to the server. The system verifies that the encrypted username and password sent by the client server are registered in the system and meet the registration information. If it is already registered information, the system provides data synchronization services. Otherwise, the user is prompted to register. 
Data localization. In the process of data transmission, data is transmitted in ICMPv6 format. Data transfer from the central database must be localized. It is converted to the format of the target database. To ensure data consistency in different services of the same system, ICMPv6 data must be parsed by the DOM parser before being written to the target database.

There are three types of users in the home-school interactive education platform based on the heterogeneous data transformation architecture: student users, teacher users, and system administrators. Students and teachers register through a mobile client application or platform, and registration information must include their school information. The system saves the user's task data in the school system, which makes it very easy for users to share information with other users in the same school.

In the overall structure diagram of the home-school interaction platform, registered users can access resources through the login interface of the platform. When the required resources are not in the system, the service system sends a request to acquire data from the central server. If the return is successful, the available resources are given to the front-end user, and a copy is saved to the local business system to update the data of the local business system. Users can also upload their own data through the system interface, and the uploaded data will be saved to the business system. The front end will periodically add and store resources to the student source database or the teacher source database of the central database system for easy access and query. Usually, the uploaded data is divided into 4 types: text, picture, video and audio. For text and picture resources, it is very convenient to save and transmit in ICMPv6 format. For audio and video, because they are too large, they can also be stored in ICMPv6 format based on Base64 encoding. However, such a copy cannot be converted to different systems at higher efficiency. The link between audio and video can only be uploaded to the network server, which can increase the efficiency of uploading and reduce the complexity of data transformation. The overall structure is shown in Figure 3.

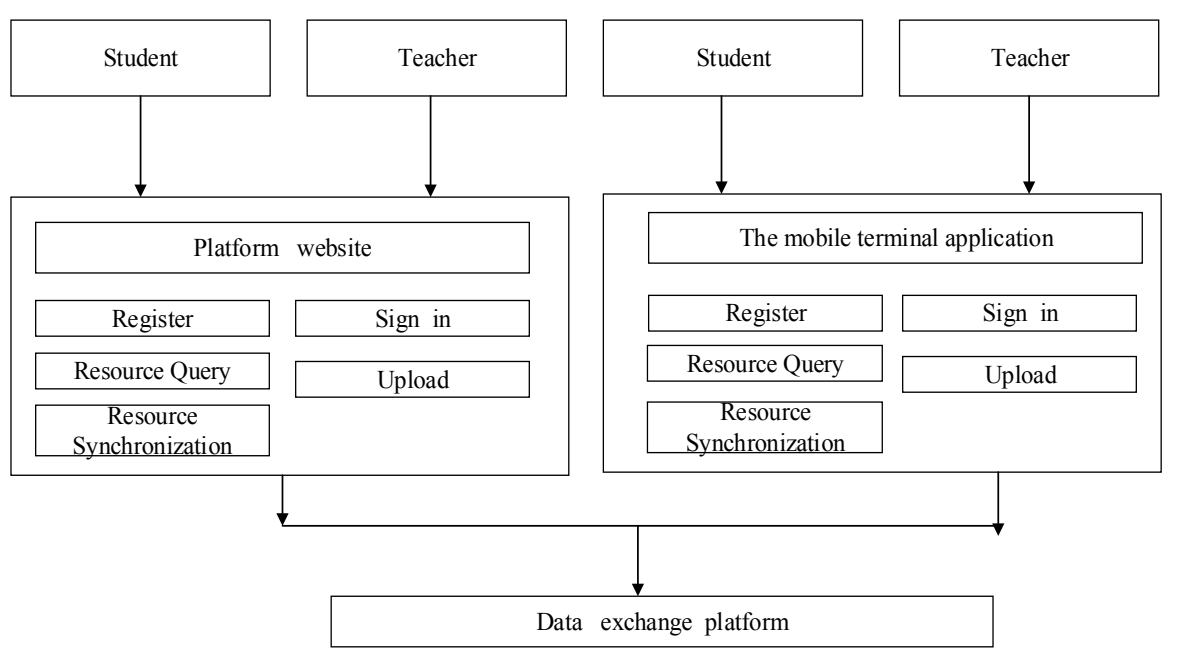

Fig. 3. Overall structure of the family-school interaction platform 


\section{$4 \quad$ Result analysis and discussion}

The architecture of the data exchange platform was studied. The heterogeneity of educational resource data leads to the problem of information silos. To solve this problem, an ICMPv6-based data exchange platform architecture is proposed, which integrates the distribution, integration and sharing of heterogeneous data resources. The architecture mainly includes design of architecture of data exchange platform and architecture of heterogeneous relational database, ICMPv6-based document conversion and data concurrency strategy and a home-school interactive education platform based on a heterogeneous data exchange platform. The architecture design provides the basis for the research and implementation of data exchange platform switching technology and application technology.

The exchange speed and security issues of the data exchange platform were studied. To improve the switching efficiency, a new parallel contention crossbar switch structure is selected for the data exchange platform. The structure was validated under five common educational resource business models. Bandwidth of link utilization at link utilization $20 \%$ is shown in Table 1.

Table 1. Bandwidth of link utilization at link utilization $20 \%$

\begin{tabular}{|c|c|c|c|c|c|}
\hline $\begin{array}{c}\text { Experiment } \\
\text { times }\end{array}$ & $\begin{array}{c}\text { Broadband } \\
\text { (link 1) }\end{array}$ & $\begin{array}{c}\text { Broadband } \\
\text { (link 2) }\end{array}$ & $\begin{array}{c}\text { Broadband } \\
\text { (link 3) }\end{array}$ & $\begin{array}{c}\text { Broadband } \\
\text { (link 4) }\end{array}$ & $\begin{array}{c}\text { Broadband } \\
\text { (link 5) }\end{array}$ \\
\hline 1 & 17.83 & 8.83 & 1.72 & 8.79 & 16.94 \\
\hline 2 & 17.32 & 8.93 & 1.78 & 8.76 & 16.91 \\
\hline 3 & 17.22 & 8.87 & 1.72 & 8.87 & 16.96 \\
\hline 4 & 17.64 & 8.89 & 1.80 & 8.94 & 16.71 \\
\hline 5 & 17.78 & 8.79 & 1.75 & 8.73 & 16.74 \\
\hline 6 & 17.56 & 8.73 & 1.75 & 8.72 & 16.90 \\
\hline Mean & 17.56 & 8.84 & 1.75 & 8.80 & 16.86 \\
\hline
\end{tabular}

The experimental results show that the new parallel contention crossbar switch can effectively improve the efficiency of the underlying data exchange of the data exchange platform. Aiming at the exchange bottleneck problem in the data exchange process, an end-to-end data traffic monitoring method is proposed. Tracert is used to record all router addresses and hop counts on the end-to-end path. The IPv6 flow label is used to extract ICMPv6 timeout packets and their corresponding router addresses and timestamps. Finally, the subtraction method is used to calculate the end-to-end bottleneck flow. Based on the new features in the IPv6 environment, this simulation experiment is to verify the asymmetric packet pair algorithm under the subtraction model. The experimental results show that the measured values obtained by the asymmetric package of the algorithm under the model are within the acceptable range, but the values are low. The stability and accuracy of the bottleneck bandwidth measurement can be maintained when $u=50 \%$ (the link is not very busy). When $u=80 \%$ (the link is very busy), the stability and accuracy of the bottleneck bandwidth measurement value are no longer maintained, and the algorithm fails. Therefore, this algorithm has both 
advantages and disadvantages. The advantage is that the location of the network bottleneck can be determined. The disadvantage is that the algorithm takes a long time to measure. Therefore, the cumulative error cannot be ignored. The results show that the link bandwidth of the bottleneck bandwidth measurement method based on the subtraction rule is stable at $20 \%$, which can effectively improve the link utilization.

\section{Conclusions}

The data exchange platform is built through a computer network. Decentralized information systems are integrated. Information and data are exchanged and shared. This mode greatly improves the utilization of information. It is the main target of the current information construction. On the basis of ensuring the interconnection of heterogeneous application systems, the database of the platform center is established to achieve data extraction, loading, and presentation. A unified data exchange center is designed. Scholars have conducted a lot of research on the functional requirements of the data exchange platform. Related technologies of the data exchange platform were explored. It includes the application research of parallel crossbar switch in data exchange platform and the research of bottleneck bandwidth measurement method in data exchange platform.

The architecture of the data exchange platform for teaching resources was studied. First, the middle layer architecture of the ICMPv6-based teaching resource data exchange platform architecture is studied. Second, the application layer architecture of the ICMPv6-based teaching resource data exchange platform architecture is studied. The architecture of the middle and application layers was studied and the theory was updated. A new network computing method was proposed. Finally, in the practical application of the family interactive education platform, the good performance of the system is verified. A data exchange and data sharing system between school and family issues was resolved. A feasible solution to the family-school interactive education platform was proposed. It provides a strong reference for the further development of the family-school interactive education platform.

With the continuous development of the Internet of Things and big data technology, the terminals and applications of the data exchange platform are gradually transformed from the traditional effective network mode to the platform that supports both the wired network and the wireless network. Therefore, based on the current research status and the research trend of big data, the future research prospects mainly include the following aspects. In terms of architecture research, the storage architecture of heterogeneous big data is further studied. A large amount of heterogeneous data is stored in a distributed disk. Heterogeneous data information is quickly acquired through wired and wireless networks. In terms of security, some security risks and features of wireless networks are discussed. The difference between wireless and wired networks is summarized. An effective solution is proposed for new security issues in wireless networks. The application of the data exchange platform was studied. According to the characteristics of wireless networks, a distributed data analysis method based on wireless networks is further proposed. The overhead of data analysis is distributed from the 
platform side to the terminal side related to the platform, thereby effectively reducing the load on the platform. It can support more services online at the same time. The application effect of the platform is improved. Simulation experiments were carried out on this intelligent guidance system and data exchange platform. In the data exchange system, there is no data set in the teaching resource data exchange platform. The two platforms will be docked in further work to implement intelligent guidance for the classified student information.

\section{$6 \quad$ References}

[1] Bartoli, G., Fantacci, R., Gei, F., Marabissi, D., \& Micciullo, L. (2015). A novel emer-gency management platform for smart public safety. International Journal of Commu-nication Systems, 28(5): 928-943 https://doi.org/10.1002/dac.2716

[2] Cai, H., Xu, B., Jiang, L., \& Vasilakos, A. V. (2017). IoT-based big data storage sys-tems in cloud computing: Perspectives and challenges. IEEE Internet of Things Jour-nal, 4(1): 75-87

[3] González, I., Calderón, A. J., Barragán, A. J., \& Andújar, J. M. (2017). Integration of Sensors, Controllers and Instruments Using a Novel OPC Architecture. Sensors, 17(7): 1512 https://doi.org/10.3390/s17071512

[4] Hakiri, A., Berthou, P., Gokhale, A., \& Abdellatif, S. (2015). Publish/subscribe-enabled software defined networking for efficient and scalable IoT communications. IEEE communications magazine, 53(9): 48-54 https://doi.org/10.1109/MCOM.2015.7263372

[5] Hussain, A., Wenbi, R., da Silva, A. L., Nadher, M., \& Mudhish, M. (2015). Health and emergency-care platform for the elderly and disabled people in the Smart City. Journal of Systems and Software, 110: 253-263 https://doi.org/10.1016/j.jss.2015.08.041

[6] Karkazis, P., Trakadas, P., Zahariadis, T., Chatzigiannakis, I., Dohler, M., Vitaletti, A., ... \& Sarakis, L. (2015). Resource and service virtualisation in M2M and IoT platforms. International Journal of Intelligent Engineering Informatics, 3(2-3): 205-224 https://doi.org/10.1504/IJIEI.2015.069897

[7] Jourjon, G., Marquez-Barja, J. M., Rakotoarivelo, T., Mikroyannidis, A., Lampropou-los, K., Denazis, S., ... \& Ott, M. (2017). FORGE toolkit: leveraging distributed sys-tems in eLearning platforms. IEEE Transactions on Emerging Topics in Computing, 5(1): 7-19 https://doi.org/10.1109/TETC.2015.2511454

[8] Xu, K., Qu, Y., \& Yang, K. (2016). A tutorial on the internet of things: from a hetero-geneous network integration perspective. IEEE Network, 30(2): 102-108 https://doi.org/10.1109/MNET.2016.7437031

\section{$7 \quad$ Author}

Wei Fang is with the Huaibei Vocational and Technical College, Anhui 235000, China (weifang3482@21cn.com).

Article submitted 26 September 2018. Resubmitted 15 October 2018. Final acceptance 29 October 2018. Final version published as submitted by the author. 